\title{
Carcinoma of the pancreas
}

\section{By K. G. Wormsley, Ninewells Hospital, Ninewells, Dundee $D D_{1} 9 S Y$}

During the past few decades, the incidence of pancreatic cancer has increased dramatically in many parts of the world. Since the incidence of other cancers has decreased, we are not faced with some general problem (such as increasing age of the population) but with a situation which points to the occurrence of factors producing cancer specifically in the pancreas. Epidemiological information of this type indicates the increasing prevalence of environmental factors which are organ-specific carcinogens and that, in turn, means that we have an objective which can be achieved - the identification of the carcinogen or carcinogens.

It has been known for many years that there are striking geographic and ethnic differences in the incidence of pancreatic cancer. It has also been shown that an increased incidence of pancreatic cancer affects workers in some industries. However, the pancreatic carcinogens have not been isolated because no technique has been available for identifying this type of organ-specific carcinogen. An unexpectedly-high incidence of pancreatic cancer has also been found in association with some other diseases, especially familial pancreatitis and ataxia telangiectasia. But perhaps the association between dietary factors and the incidence of pancreatic cancer provides the most interesting pointer to aetiological factors implicated in pancreatic carcinogenesis. Thus, it has been shown that there is a sort of 'dose-response' relation between the incidence of pancreatic cancer and the intake of dietary fat.

We can develop an aetiological hypothesis from this finding. Dietary fat is known to be a powerful stimulant of the release of the hormone cholecystokinin (CCK) from the small intestine. CCK not only stimulates pancreatic exocrine secretion but is also a powerful pancreatic growth-promoting hormone. It therefore seems possible that greater amounts of dietary fat, by releasing $\mathrm{CCK}$ in greater amounts, stimulate excessive pancreatic growth which, in turn, leads directly to autonomous growth and cancer or acts indirectly by sensitizing to, and promoting the effects of, pancreatic carcinogens. The hypothesis has been tested in rats by using exogenous CCK, given by injection to the animals. Two of a series of rats treated in this way for 6 months developed pancreatic cancer. A better method for ensuring continuous pancreatic stimulation with abnormally-large amounts of $\mathrm{CCK}$ is provided by dietary manipulation. If rats are given diets containing as little as $50 \mathrm{~g}$ raw soya-bean flour $/ \mathrm{kg}$ diet, the small intestine of these animals releases large amounts of CCK which, in turn, stimulates continuing growth of the pancreas. The growth at first results in general enlargement of the gland, but soon foci of abnormal growth occur, which become autonomous adenomata and then 
carcinomas. About one in six rats given raw soya-bean flour develop pancreatic cancer. Even more interestingly, a diet containing raw soya-bean flour potentiates the effects of a subthreshold dose of pancreatic carcinogen, just as CCK potentiates the bicarbonate-secretory response of the pancreas to a subthreshold dose of secretin. In this role, raw soya-bean flour-containing diets act to promote the effect of a genotoxic (initiating) carcinogen. By indicating, in general, how pancreatic cancer may develop in response to dietary factors, the finding may have specific implications for the development of human pancreatic cancer. In addition, it is clear that the rat given raw soya-bean flour is a very sensitive model that can be used for screening environmental chemicals for potential pancreatic carcinogenic effects.

In addition to the promoting effects of raw soya-bean flour, it has also been shown recently that a diet containing unsaturated fat promotes the development of pancreatic cancer in rats when these animals have been exposed to a pancreatic carcinogen. This effect is not as organ-specific as the effect of raw soya-bean flour, since unsaturated fats also promote the development of cancer of breast, skin, colon, etc.

As well as establishing the cancer-promoting effects of dietary manipulation, it has also been possible to show that the development of pancreatic cancer is promoted by other conditions which result in proliferation of the pancreatic acinar cells. Thus, pancreatic carcinoma develops unexpectedly frequently if subthreshold amounts of pancreatic carcinogen (amounts which by themselves do not produce pancreatic cancer) are administered to rats after partial pancreatectomy or to rats recovering from acute pancreatitis. It seems that pancreatic regeneration after injury also promotes the effect of the pancreatic carcinogen, just as the dietary stimulus to growth does.

In summary, it seems that pancreatic cancer develops when the pancreas has been stimulated to replication as a result of a stimulus to regeneration or growth, if at that time the replicating tissue is exposed to an initiating carcinogen. 